\title{
FUNCIONALIDAD FAMILIAR, CONDUCTAS EXTERNALIZADAS Y RENDIMIENTO ACADÉMICO EN UN GRUPO DE ADOLESCENTES DE LA CIUDAD DE BOGOTÁ*
}

\author{
FAMILY FUNCTIONALITY, EXTERNALIZED BEHAVIOR AND ACADEMIC \\ ACHIEVEMENT IN A GROUP OF ADOLESCENTS BOGOTÁ CITY* \\ JAIME HumberTo MORENO MÉNDEZ** \& LAURA CAMILA CHAUTA ROZO*** \\ UNIVERSIDAD DE SAN BUENAVENTURA, SEDE BOGOTÁ, - COLOMBia \\ FECHA DE RECEPCIÓN: 5/09/2011 • FECHA DE ACEPTACIÓN: 12/12/2011
}

\begin{abstract}
Resumen
El presente trabajo investigativo tuvo como objetivo describir la asociación entre la funcionalidad familiar, las conductas externalizadas y el rendimiento académico en un grupo de adolescentes de un colegio distrital de la localidad de Usaquén de la ciudad de Bogotá. El estudio fue de corte empírico-analítico de tipo descriptivo y un método de asociación, empleando como instrumentos la lista de chequeo de la conducta infantil de Achenbach y Edelbrock, los tres cuestionarios, el de autorreporte (YSR), el cuestionario de padres (CBCL) y el cuestionario de profesores (TRF), el APGAR familiar y el boletín académico. Los participantes fueron adolescentes entre 12 y 16 años de edad, sus respectivos padres y profesores. Dentro de los resultados encontrados se estableció que la mayoría de los adolescentes reportan una disfunción familiar moderada y un rendimiento académico medio. Igualmente se encontró que la mayoría de ellos no presenta conductas externalizadas aunque hay un porcentaje de ellos que sí las presenta, especialmente según lo reportado por los profesores.
\end{abstract}

Palabras clave: funcionalidad familiar, conductas externalizadas, rendimiento académico, adolescentes.

\begin{abstract}
This investigative work has as objective to describe the association between family functionality, externalized behaviors and academic achievement by a group of adolescents of school district in the town of Usaquén of Bogotá. This work was of empirical-analytical and descriptive with an association method, using instruments such as the checklist of child behavior of Achenbach and Edelbrock, The three questionnaires, self-report (YSR), the parent questionnaires (CBCL) and teachers' questionnaire (TRF), the family APGAR and the academic journal. Participants were adolescents aged 12 to 16 years old, their parents and teachers. Among the results established that the majority of adolescents reported family dysfunction moderate and average academic performance. It was also found that most of them haven 't externalized behavior, although there is a percentage of them that if present, especially as reported by teachers.
\end{abstract}

Keywords: Family functionality, externalized behavior, academic achievement, adolescents.

* Investigación realizada en el Grupo Avances en Psicología Clínica y de la Salud, de la Facultad de Psicología de la Universidad de San Buenaventura, sede Bogotá. ** Magíster en Psicología Clínica. Investigador principal. Correspondencia: jaimehm@yahoo.com

***Psicóloga. Coinvestigadora. Correspondencia: chautykmy23@gmail.com

| PSYCHOL. AV. DISCIP. | BOGOTÁ, COLOMBIA | VOL. 6. | N. ${ }^{\circ}$ 1. | P. 155-166 | ENERO-JUNio | 2012 | ISSN 1900-2386 | 


\section{Introducción}

Los estudios a través del tiempo han demostrado que la familia es un componente vital en la formación de los individuos, además de constituir la sociedad más antigua de la historia, lo que la convierte en el primer vínculo de socialización que posee cualquier ser humano. Varias son las investigaciones que se han realizado en torno a esta, las cuales favorecen positivamente el funcionamiento de la misma.

La familia es un sistema que a su vez se compone de subsistemas; el primero de ellos el conyugal (papá y mamá), el segundo paterno-filial (padres e hijos) y el fraternal (hermanos). Todas las familias tienen características que las pueden hacer distintas o similares a otras, algunas características tipológicas son: la composición (nuclear, extensa o compuesta) y el desarrollo (tradicional o moderno) (Mendoza, Soler, Sainz, Gil, Mendoza \& Pérez, 2006).

Para poder entender esta serie de sistemas es importante conocer acerca del ámbito familiar en el cual existen diversas reglas que restringen el comportamiento de cada persona respecto a su entorno, un factor importante a tener en cuenta es el espacio o lugar donde se encuentra ubicada la residencia (urbana, suburbana o rural), la ocupación de los miembros de la familia (campesino, empleado o profesional), la forma en la cual se encuentra integrada (integrada, semiintegrada o desintegrada), entre otras, de igual manera existen otras características que son indispensables de conocer, las cuales son útiles para determinar el grado de funcionalidad familiar.

La Funcionalidad Familiar o una familia funcional, es aquella que logra promover el desarrollo integral de sus miembros así como un estado de salud favorable en ellos donde los miembros de la misma perciben el funcionamiento familiar manifestando el grado de satisfacción con el cumplimiento de los parámetros básicos de la función familiar, como son, adaptación, participación, ganancia o crecimiento, afecto y recursos (Smilkstein, 1978; Paz, 2007).

Relacionada con la funcionalidad, se encuentra la jerarquía entendiéndola como el nivel de autoridad que gobierna en la organización familiar y que pueden ser, autoritaria, indiferente negligente, indulgente, permisiva o recíproca con autoridad, los límites que representan las reglas que delimitan a las jerarquías y que pueden ser, claros, difusos o rígidos y la comunicación que no es más que la forma en que la familia se expresa entre sí (Solis, Huerta, Vásquez, Alfaro, Sánchez \& Hernández, 2006).

Según Forero, Avendaño, Durán, Duarte y Campo (2006), la familia es una unidad biopsicosocial y una de sus funciones más importantes es contribuir a la salud de todos sus miembros, por medio de la transmisión de creencias y valores de padres a hijos, así como el apoyo brindado.

Así, los niños adquieren nuevas experiencias con el paso del tiempo, esto les permite desarrollarse adecuadamente mientras avanzan hacia la etapa adolescente, al llegar a esta, algunos presentan dificultades en su comportamiento, cambios a nivel cognitivo, físico y psicológico lo cual convierte al adolescente en un integrante que genera inestabilidad al interior de la estructura familiar y puede generar malestar en cuanto a su funcionalidad debido a los cambios tanto emocionales como comportamentales que está experimentando el individuo.

Dentro de la familia, se pueden evidenciar tanto factores de riesgo como de protección hacia el adolescente, los factores de riesgo hacen referencia a las características internas y/o externas del individuo, que aumentan la probabilidad o predisposición de que se produzca un determinado fenómeno (Clayton, 1992). Asimismo, la falta de apoyo, el trato violento, la deserción escolar y la poca estancia en el hogar que experimentan los adolescentes, forman parte de dichos factores mientras que las relaciones familiares basadas en la comunicación se convierten en un factor protector de primer orden, y pueden disminuir las influencias adversas que se presentan en el contexto social.

Así se tiene que los factores de Protección, son aquellos factores psicológicos o sociales que modifican la acción de un factor de riesgo para desestimular o evitar la aparición de algún problema en las personas. Además de ser los recursos individuales o condiciones sociales que atenúan o neutralizan el impacto del riesgo (Clayton, 1992). En algunos estudios se ha encontrado que los factores de riesgo anteriormente mencionados son más evidentes cuando existe poco interés y baja comunicación por parte de los padres frente al grupo de pares de sus hijos, igualmente, el tener malestar físico y psicológico; por lo que determinados ambientes familiares, la falta de 
comunicación o las relaciones distantes pueden favorecer dichas conductas en los adolescentes (Rodrigo, Máiquez, García, Mendoza, Rubio, Martínez \& Martín, 2004).

Las relaciones distantes aumentan la probabilidad de conductas tales como el consumo descontrolado de sustancias psicoactivas (drogas, alcohol, tabaco), delincuencia, deserción escolar y relaciones sexuales precoces; mientras que las relaciones afectivas aumentan significativamente la seguridad, la autoestima, el autoconcepto y factores determinantes en el desarrollo de destrezas emocionales, cognitivas y sociales del adolescente (Silva y Pillón, 2004). Por otra parte, los adolescentes que pertenecen a familias con buena comunicación, un grado alto de adaptabilidad, una fuerte vinculación emocional entre sus miembros y mayor satisfacción familiar, perciben mayor apoyo en cuanto a sus relaciones personales significativas (Jiménez, Musitu \& Murgui, 2005).

Cuando las relaciones familiares no son armónicas surgen dificultades de adaptación y problemas de comportamiento en los adolescentes que a su vez impactan recíprocamente en la funcionalidad familiar.

Existe suficiente evidencia empírica que permite establecer dos grandes grupos de conductas o características en las desviaciones del comportamiento infantil y adolescente. Uno de estos grupos ha sido llamado conducta externalizante, subcontrolada o desorden de conducta, que es en general aquella que se caracteriza por una carencia de control y sintomatología hiperactiva o agresiva, a este patrón se asocian las peleas, las rabietas, la desobediencia y la destrucción. El segundo grupo ha sido llamado internalizante, sobrecontrolado o ansiedadaislamiento, es decir, aquellas conductas más pasivas que involucran aislamiento, ansiedad, depresión y preocupaciones somáticas (Wicks-Nelson, 2000).

Achenbach y Rescorla (2001), en diversos estudios realizados han identificado ocho escalas denominadas de banda estrecha o síndromes de primer orden y tres dimensiones de segundo orden o banda ancha. Los síndromes de primer orden son depresión/retraimiento, quejas somáticas, depresión/ansiedad, problemas sociales, problemas de pensamiento, problemas de atención, conducta agresiva y conducta delictiva. Las dimensiones de segundo orden hacen referencia a una dimensión Internalizante, una Externalizante y otra Mixta. La dimen- sión Internalizante implica la vivencia de tensión psicológica en el propio sujeto, síntomas de angustia, depresión y estados alterados de ánimo, y agruparía los síndromes depresión/ retraimiento, quejas somáticas y depresión/ ansiedad. La dimensión Externalizante está relacionada con síntomas de agresividad, déficit de atención, hiperactividad, conducta desorganizada, y agruparía los síndromes de conducta delictiva y conducta agresiva. Finalmente, la tercera dimensión de tipo Mixto agrupa los problemas de pensamiento, sociales y de atención que no se han logrado asociar de una manera clara en ninguna de las dimensiones anteriores.

Los comportamientos internalizados y externalizados constituyen un conjunto de síntomas, no obstante, estos se diferencian unos de otros según dichas conductas, en el caso de las primeras, es difícil detectar la presencia de alguna dificultad, debido a que aparentemente son niños tranquilos y adaptados a su entorno, sin embargo, son personas que interiormente están enfrentando preocupaciones y pensamientos poco adecuados (distorsiones cognitivas).

A continuación se realizará una revisión sobre cada uno de los dos grupos, comenzando por el externalizante, que se caracteriza por comportamientos de agresividad y ruptura de normas, que son dirigidos hacia el exterior y afectan a otras personas, asimismo, se encuentran clasificados en dos grupos de síndromes, el primero de ellos llamado conducta o comportamiento de ruptura de reglas o normas, y el segundo, denominado conducta o comportamiento agresivo; de igual manera plantean un grupo de comportamientos presentes dentro de estas conductas tales como, exigir mucha atención, agredir, chillar, amenazar, fastidiar, ser irritable, envidioso, desobediente y tirano entre otros (Achenbach \& Rescorla, 2001).

Dentro de las teorías mencionadas se encuentra un punto de discusión en Graber (2004), quien propone que los comportamientos externalizados son aquellos que se encuentran desviados de la norma; mientras que Barber (2002), señala que estos comportamientos se caracterizan por estar guiados hacia el exterior, por ejemplo, la agresividad, los robos y las mentiras, entre otros, planteando así que estos comportamientos no son adaptativos ante la sociedad, ya que generan daño o dolor a otras personas. 
En Colombia, Ballesteros (1995), realizó un estudio centrado en el conflicto entre padres, y encontró que este genera en los hijos conductas tales como ansiedad, temor y enojo, lo cual impulsa al hijo a tener reacciones comportamentales que pueden ser adaptativas o desadaptativas ya sea de tipo externalizante o internalizante.

Igualmente, desde una perspectiva biopsicosocial, los comportamientos tanto internalizados como externalizados son factores que constituyen vulnerabilidad o riesgo de un amplio número de trastornos y síntomas, los cuales en un futuro, podrían estar íntimamente ligados con problemas de personalidad u otro tipo de psicopatología. (Zuckerman y Krueger, 2000; citado por Maestre, Moya, Edo, Mezquita, Rupérez \& Villa, 2004).

Otra de las investigaciones que se han realizado con el fin de esclarecer y ampliar el panorama sobre los problemas externalizados y su relación con el entorno familiar, es la de Medina, Navarro, Martinena, Baños, Vicens-Vilanova, Barrantes-Vidal, Subirá y Obiols (2007). Mediante un estudio realizado en Barcelona, España, con 160 participantes dentro de los cuales se encontraban, padres, maestros y adolescentes de los 13 a los 16 años, se buscaba explorar los patrones de acuerdo entre informantes sobre los problemas conductuales presentes en adolescentes de población general e igualmente en los casos en los cuales no se presentaba acuerdo, además de analizar el grado de discordancia entre cada par de informantes para cada subescala, cabe resaltar que el instrumento que se utilizó para dicho estudio fue la escala de alteraciones conductuales de Achenbach, (Youth Selft-Report, Child Behavior Checklist 4-18, Teacher's Report Form).

Los resultados arrojados por esta investigación demuestran que la concordancia entre informantes era baja, pues los padres comunicaban el mayor índice de alteraciones seguidos de los estudiantes y los maestros, esto podría deberse a que por ejemplo los maestros se encuentran más acostumbrados a estar con los adolescentes, y por este motivo consideran algunas alteraciones conductuales en el adolescente como normales. Para los padres, las conductas internalizadas son más preocupantes, puesto que estas conductas adolescentes suelen ser más perturbadoras en el contexto familiar, finalmente son los adolescentes quienes reportan mayores conductas externalizadas lo cual podría explicarse por la deseabilidad/indeseabilidad social que experimentan estos a su grupo de pares.
Ahora bien, la presencia de conductas externalizadas, perjudica el desarrollo del adolescente a nivel familiar, y su proceso de aprendizaje, este último se hace evidente en el rendimiento escolar, el cual hace referencia al nivel de logro que es capaz de alcanzar un estudiante dentro de un ambiente escolar o de alguna asignatura en particular, este puede ser valorado a través de evaluaciones pedagógicas, dichas evaluaciones son aquellas estrategias que utiliza el educador en el transcurso del año escolar, lo cual le permite obtener información necesaria para evaluar el logro (Sattler, 2003).

Jiménez (2000), define el rendimiento académico como el nivel de conocimientos que demuestra el estudiante en un área o materia, el cual se compara tanto con la edad como con el nivel académico. Sin embargo, el rendimiento académico debería entenderse a partir de los procesos de evaluación, pero la medición y evaluación simple del rendimiento alcanzado por los alumnos, no genera por si mismo las pautas necesarias para el mejoramiento de la calidad educativa.

Para entender el rendimiento académico desde la perspectiva evaluativa, es necesario tener en cuenta, no solo el desempeño individual, sino también la influencia del grupo de pares, el aula o el propio contexto educativo (Edel, 2003).

Portellano (1989) propone dos tipos de rendimiento, el rendimiento suficiente y el rendimiento satisfactorio, el primero, se utiliza como una medida de aptitud pedagógica que evalúa por medio de las calificaciones escolares, este tipo de rendimiento se encuentra en función de los conocimientos escolares que se adquieren; en el segundo caso, este se menciona cuando se toma como punto de referencia la capacidad intelectual, teniendo en cuenta las relaciones que se dan entre capacidades intelectuales y aprovechamiento de las mismas.

Cominetti y Ruiz (1997), desarrollaron un estudio denominado "Algunos factores del rendimiento: las expectativas y el género", dentro de los resultados obtenidos por esta investigación, se encuentra que las expectativas de la familia, docentes y alumnos en cuanto a los logros de aprendizaje tiene gran interés, puesto que pone al descubierto un conjunto de prejuicios, actitudes o conductas que pueden ser ventajosas o desventajosas en cuanto al desarrollo escolar y sus resultados. Asimismo, el rendimiento de los alumnos es mejor cuando los 
docentes manifiestan un nivel de desempeño y comportamiento adecuado.

A pesar de esto, todas aquellas personas que se encuentran relacionadas con el ámbito escolar se preocupan excesivamente por las notas que obtienen los estudiantes, principalmente cuando dichas notas son "malas", por lo cual buscan mejorar el rendimiento en cuanto al aprendizaje que tienen los alumnos; la familia por su parte se siente angustiada cuando sus hijos o hijas obtienen notas no tan satisfactorias. Es por esta razón que las autoridades académicas realizan grandes esfuerzos para modificar los planes de estudio con el fin de obtener aumentos en el nivel de rendimiento (Antoni, 2006).

Sin embargo, ya que una de las variables mas empleadas por parte de los maestros para acercarse al rendimiento académico son las calificaciones, es importante tener en cuenta el estudio realizado por Cascón (2000), quien atribuye la importancia de estas a dos razones principales, la primera, se refiere a la obtención de un sistema educativo eficiente y eficaz que logre proporcionar a los estudiantes un marco ideal en el cual puedan ser desarrolladas sus potencialidades, la segunda, el indicador de nivel educativo ha sido y probablemente seguirá siendo las calificaciones escolares, las cuales se obtienen a partir de evaluaciones o exámenes, donde el estudiante debe demostrar sus conocimientos acerca de un área o materia que el sistema educativo considera necesaria o por lo menos suficiente para su desarrollo adecuado como miembro activo de la sociedad.

Ahora bien, teniendo en cuenta lo anterior, en un estudio realizado en Envigado, Antioquia, con 451 adolescentes de grado noveno de instituciones oficiales, se encontró que el estilo parental afecta significativamente el desempeño académico, puesto que un estilo de autoridad indulgente es decir permisivo genera un desempeño menor a nivel académico, mientras que el estilo rígido, es decir autoritario, genera un nivel mayor en cuanto al desempeño académico (Tilano, Henao \& Restrepo, 2008).

Asimismo, Girod (1984, citado por Antoni, 2006), establece cuatro factores para el éxito escolar, el primero de ellos es el estudiante y su trayectoria escolar, como medio para conocer sus debilidades académicas, el segundo, las condiciones bajo las cuales se están generando la enseñanza, tales como planes de estudio y actividades académicas, el tercero, las características familiares, como una manera de establecer vínculos de acompañamiento académico por parte de los padres, y finalmente, las características comportamentales del estudiante, puesto que el desempeño de los estudiantes va ligado a la manera como se comporta este dentro del establecimiento educativo y frente a cada una de las asignaturas que toma.

Como se puede apreciar, de todo lo explicitado anteriormente, diversos estudios han encontrado que el comportamiento y el rendimiento académico en los adolescentes guarda una importante relación con la dinámica familiar, por ello es importante profundizar y analizar cómo se presenta dicha relación en población en el contexto local con miras a tener datos que sirvan para futuras acciones de prevención. Por esta razón, el objetivo de la presente investigación fue identificar la asociación entre el nivel de funcionalidad familiar, las conductas externalizadas y el rendimiento académico en un grupo de adolescentes de un colegio distrital de la localidad de Usaquén en la ciudad de Bogotá.

\section{Método}

\section{Diseño}

Este estudio fue de corte empírico-analítico de tipo descriptivo y un método de asociación, ya que se buscaba describir la relación existente entre la funcionalidad familiar, las conductas externalizadas y el rendimiento académico. Este estudio utilizó el método de asociación debido a que este trabaja con variables nominales u ordinales (Briones, 1998), teniendo en cuenta que la variable rendimiento académico se encuentra dentro de la escala ordinal, es necesario llevarlo a cabo de esta manera.

\section{Participantes}

Dentro de la investigación participó un grupo de 63 adolescentes, 34 de ellos de sexo femenino y los 29 de sexo masculino con edades entre los 12 y 16 años de edad de los grados octavo y noveno en la jornada mañana y tarde seleccionados al azar, un grupo de 63 padres de familia correspondientes a cada uno de los adolescentes de la muestra y 4 profesores de un colegio distrital ubicado en la localidad de Usaquén en la ciudad de Bogotá.

\section{Instrumentos}

Lista de chequeo de la conducta infantil de Achenbach y Rescorla (2001), formato de autorreporte: fue diseñado para obtener información acerca de las competencias y proble- 
mas de jóvenes de 11 a 18 años. Se basa en el supuesto de que los jóvenes tienen un conocimiento mayor y completo de sus propias conductas a través de diferentes situaciones y pueden expresar opiniones de su propio funcionamiento. El autorreporte es calificado sobre un perfil, en el cual se incluyeron las dos escalas del formato anterior de padres, la primera es la de competencia, con ítems sobre compromisos en deportes, otras actividades recreativas y organizacionales, número de amigos y contactos con ellos y calidad del desempeño individual. La segunda escala es la de problemas de conducta, diseñada para identificar problemas específicos y síndromes de problemas que coocurren en poblaciones clínicas. La correlación de Pearson para el grupo de 11 a 14 años fue de .77 y para el de 15 a 18 años, de .89 (Achenbach \& Rescorla, 2001).

Lista de chequeo de la conducta infantil de Achenbach y Edelbrock, formato de padres: fue diseñado en respuesta a la necesidad de procedimientos estandarizados para registrar los comportamientos relevantes en los niños de 4 a 16 años. El instrumento tiene dos escalas principales, la de competencia social (20 ítems) sobre la cantidad y calidad de la participación de los niños en deportes, pasatiempos, juegos, organizaciones, labores y amistades, así como el funcionamiento escolar, y la de problemas (118) ítems. El Indice de confiabilidad es de .985 para los ítems de problemas y de .978 para los de competencia.

Lista de chequeo de la conducta infantil de Achenbach y Edelbrock, formato de profesores: fue diseñado a partir del CBCL para ser respondido por profesores que conozcan a los niños y jóvenes por un tiempo mínimo de dos meses. Este formato también incluye las dos escalas de los demás, la de competencia (desempeño escolar y funciones adaptativas) y la de problemas. El Indice de confiabilidad es de .59 para la de externalización y de 46 para la de internalización.

APGAR Familiar: es un instrumento diseñado y desarrollado por Smilkstein (1978), para aproximarse de una manera objetiva a la función familiar del paciente. Consiste en una tabla con cinco enunciados que hacen referencia a las características básicas que hacen que la familia sea funcional y armónica, estos enunciados hacen referencia a: Adaptación, Participación, Crecimiento, Afecto y Resolución. Cada uno de los enunciados se evalúa en una escala de cinco opciones: Siempre $=4$, Casi siempre $=$ 3; Algunas veces $=2$; Casi nunca $=1$; Nunca $=0$. La interpretación del resultado es:
Buena función familiar: 18-20 puntos

Disfunción familiar moderada: 10-17 puntos

Disfunción familiar severa: 9 puntos o menos

Boletín académico: Las notas que se manejan en el colegio son agrupadas de la siguiente manera, de 2.0 a 2.9 se estableció un nivel bajo de rendimiento académico, de 3.0 a 3.9 se estableció un nivel medio de rendimiento académico y de 4.0 a 4.9 se estableció un nivel alto de rendimiento académico.

El rendimiento suficiente, se utiliza como una medida de aptitud pedagógica que evalúa a los estudiantes por medio de las calificaciones escolares, este tipo de rendimiento se encuentra en función de los conocimientos escolares que se adquieren (Portellano, 1989).

\section{Procedimiento}

Fase de inicio: se realizó una solicitud por escrito a la institución educativa con el fin de pedir su aprobación para realizar la investigación, además de esto se realizó el muestreo de la población para obtener el $\mathrm{N}$ con el cual se trabajó.

Fase de implementación: se realizó la aplicación del formato de autorreporte, formato para padres y formato para profesores de la lista de chequeo de la conducta infantil de Achebnach y Edelbrock, y del APGAR familiar, explicando en términos simples el objetivo de la investigación, finalmente se revisaron los boletines escolares con el fin de obtener información acerca de las calificaciones de los estudiantes, luego de aplicar los instrumentos se procedió a organizar y analizar la información.

Fase de finalización: a partir de los resultados obtenidos, se realizó una discusión contrastando dichos resultados con la revisión teórica.

\section{Consideraciones éticas}

Se realizó la firma del consentimiento informado por parte de los padres de familia. De igual manera se realizó la firma de los asentimientos informados correspondientes por parte de los adolescentes, luego de esto se procedió a la aplicación de los instrumentos, al finalizar la fase de aplicación se realizó un taller con los adolescentes para retroalimentar el trabajo realizado con ellos.

\section{Resultados}

Para el análisis de los datos se utilizó el programa estadístico SPSS. Se realizaron los diferentes análisis, las 
variables fueron de corte tanto nominal como ordinal y fueron analizadas de manera cuantitativa. A continuación se presentaron los resultados obtenidos por el estudio, estos se presentaron en dos momentos diferentes, el primero, es el análisis descriptivo de los datos y el segundo, es el análisis por asociación de las variables. Para esta investigación se tomó como puntaje significativo del chicuadrado de Pearson aquellos puntajes inferiores a .05.

En la tabla 1 se presentan los porcentajes referidos a las variables descriptivas. En cuanto a los niveles de funcionalidad familiar de los adolescentes, el 39\% de ellos tiene un núcleo familiar funcional, el 44\% presenta disfuncionalidad moderada y finalmente el 15\% presenta disfuncionalidad severa.

Según el autorreporte de los adolescentes sobre las conductas externalizadas (YSR), el 73\% no presentan pro- blemas, mientras que el 27\% restante presenta conductas externalizadas ya sea en un rango de riesgo o clínico.

El reporte que hacen los padres sobre las conductas externalizadas de sus hijos adolescentes (CBCL), es que el 74,6\% no presentan problemas de conductas externalizadas y el 25,4\% restante sí las evidencia.

De acuerdo con lo reportado por los profesores sobre las conductas externalizadas de los estudiantes (TRF), el 30\% de los adolescentes no presentan conductas externalizadas mientras que el 70\% restante sí presenta este tipo de comportamientos.

En relación con el rendimiento académico, el $15,9 \%$ se encuentra en un nivel alto, el 71,4\% en un nivel medio y finalmente el 12,7\% en un nivel bajo.

En la tabla 2 se encuentran los porcentajes entre la funcionalidad familiar y las conductas externalizadas, que

Tabla 1. Distribución porcentual de las variables descriptivas

\begin{tabular}{lc}
\hline \hline & Porcentaje \\
\hline Funcionalidad Familiar en Adolescentes & 39,7 \\
Funcional & 44,4 \\
Disfunción moderada & 15,9 \\
Disfunción severa & \\
Conductas externalizadas en adolescentes (YSR) & 73,0 \\
No problema & 27,0 \\
Sí problema & \\
Conductas externalizadas en adolescentes (CBCL) & 74,6 \\
No problema & 25,4 \\
Sí problema & \\
Conductas externalizadas en adolescentes (TRF) & 30 \\
No problema & 70 \\
Sí problema & \\
Rendimiento académico en adolescentes & 15,9 \\
Alto & 71,4 \\
Medio & 12,7 \\
Bajo & \\
\hline \hline
\end{tabular}

Tabla 2. Asociación entre la funcionalidad familiar y las conductas externalizadas

\begin{tabular}{llcc}
\hline \hline & & \multicolumn{2}{c}{ Conductas externalizadas } \\
\cline { 3 - 4 } & & No problema & Sí problema \\
\hline \multirow{2}{*}{ Funcionalidad familiar } & Funcional & $28,60 \%$ & $11,10 \%$ \\
& Disfunción moderada & $33,30 \%$ & $11,10 \%$ \\
& Disfunción severa & $11,10 \%$ & $4,80 \%$ \\
\hline \hline
\end{tabular}


Tabla 3. Asociación entre la funcionalidad familiar y el rendimiento académico

\begin{tabular}{lllll}
\hline \hline & & \multicolumn{3}{c}{ Rendimiento académico } \\
\cline { 3 - 4 } & & Alto & Medio & Bajo \\
\hline \multirow{2}{*}{ Funcionalidad familiar } & Funcional & $4,8 \%$ & $28,6 \%$ & $6,3 \%$ \\
& Disfunción moderada & $9,5 \%$ & $30,2 \%$ & $4,8 \%$ \\
& Disfunción severa & $1,6 \%$ & $12,7 \%$ & $1,6 \%$ \\
\hline \hline
\end{tabular}

Tabla 4. Distribución de las conductas externalizadas y el rendimiento académico

\begin{tabular}{ccccc}
\hline & & \multicolumn{3}{c}{ Rendimiento académico } \\
\cline { 2 - 4 } Conductas externalizadas & Alto & Medio & Bajo \\
\cline { 2 - 5 } & No problema & 8 & 32 & 6 \\
& & $12,7 \%$ & $50,8 \%$ & $9,5 \%$ \\
& Sí problema & 2 & 13 & 2 \\
& & $3,2 \%$ & $20,6 \%$ & $3,2 \%$ \\
\hline \hline
\end{tabular}

Tabla 5. Asociación entre las conductas externalizadas y el soporte de amigos

\begin{tabular}{lcccc}
\hline \hline & & \multicolumn{3}{c}{ Soporte amigos } \\
\cline { 3 - 4 } & & Funcional & Disfuncional moderado & Disfuncional severa \\
\hline Conductas & No problema & 28 & 17 & 1 \\
externalizadas & & $44,40 \%$ & $27,00 \%$ & $1,60 \%$ \\
& Sí problema & 12 & 4 & 1 \\
& & $19,00 \%$ & $6,30 \%$ & $1,60 \%$ \\
\hline \hline
\end{tabular}

con una significancia de .94 , se establece que no existe un nivel significativo de asociación entre estas dos variables.

En la tabla 3 se presentan los resultados de la asociación entre las variables funcionalidad familiar y rendimiento académico, donde en un nivel funcional, el 4.8\% de los adolescentes presentan un nivel alto de rendimiento académico, el 28,6\% se encuentran en un nivel medio y un $6,3 \%$ en un nivel bajo. En cuanto a la disfunción moderada, el $9.5 \%$ se encuentran en un nivel alto de rendimiento académico, el 30,2\% en un nivel medio y un $4,8 \%$ en un nivel bajo. Finalmente, en la disfunción severa, el 1,6\% se encuentra en un nivel alto, el 12,7\% en un nivel medio y el 1,6\% en un nivel bajo.

Como se puede observar en la tabla 4 , con una significancia de .84 se establece que no existe un nivel significativo de asociación entre las conductas externalizadas y el rendimiento académico.
En la tabla 5 Se encuentra la asociación entre las variables conductas externalizadas y soporte de amigos, y con una significancia de .49 se establece que no existe una asociación significativa entre estas dos variables.

\section{Discusión}

El objetivo del estudio fue identificar la asociación entre el nivel de funcionalidad familiar, las conductas externalizadas y el rendimiento académico en un grupo de adolescentes de un colegio distrital de la localidad de Usaquén en la cuidad de Bogotá. Al hacer el análisis de los resultados se evidenció que la mayoría de los adolescentes reportan una disfunción familiar moderada y un rendimiento académico medio. Igualmente se encontró que la mayoría de ellos no presenta conductas externalizadas aunque hay un porcentaje de ellos que sí las pre- 
senta, especialmente según lo reportado por los profesores. Por otro lado, se concluyó que no existe un grado significativo de asociación entre el nivel de funcionalidad familiar, las conductas externalizadas y el rendimiento académico siendo cada una de estas variables de vital importancia en el desarrollo de los adolescentes.

De acuerdo a los resultados obtenidos, se logró establecer que la mayoría de la población adolescente posee un nivel disfuncional moderado dentro del núcleo familiar y solo una pequeña parte de la población se encuentra en un nivel funcional dentro del núcleo familiar, por su parte las mayoría de los adolescentes reportan que el soporte de amigos se encuentra en un nivel funcional, y solo una pequeña parte de la población adolescente reportan niveles disfuncionales en esta escala, esto puede deberse a que en esta etapa los adolescentes poseen redes de compañeros, lo cual les permite desarrollar habilidades sociales, dichas redes evalúan las habilidades, características, rasgos, aspecto personal entre otros a través de la comparación social, además de esto estas redes de compañeros le permiten al adolescente forjar nuevas amistades basadas en los intereses y actividades comunes (Craig, 1997).

De la misma manera y como parte de los cambios del desarrollo que presenta el adolescente se ponen a prueba las habilidades, sentimientos y valores que han sido inculcados en este por sus padres, en esta etapa del desarrollo, el desinterés de los cuidadores frente al grupo de pares de sus hijos, acompañado de malestar físico y psicológico dentro del ambiente familiar puede generar la aparición de diferentes problemáticas (Rodrigo et ál., 2004).

De acuerdo a los resultados se puede establecer que el soporte de amigos se convierte en un factor protector para los adolescentes disminuyendo la aparición de conductas externalizadas.

Por otro lado, dentro de los resultados, se evidencia que un alto porcentaje de los adolescentes no reporta conductas externalizadas, y solo una porción de la muestra las presenta, esto puede deberse a que en la adolescencia los individuos se encuentran en un proceso de construcción social a nivel conceptual, donde se presentan cambios a nivel del rol social en grupos de referencia, la percepción del cuerpo, la identidad y la imagen sexual, las expectativas para el comportamiento independiente y la madurez, por lo cual los adolescentes no perciben estas conductas como problemáticas (Alsaker,1995 citado por Lerner \& Steinberg, 2004). A diferencia de lo hallado en el presente estudio, Lozano y García (2000), encontraron en la provincia de Jaén (España), que tanto mujeres como hombres presentaban conductas externalizadas de corte agresivo y delictivo. De la misma manera discrepa del estudio realizado en Barcelona (España) por Medina, Navarro, Martinena, Baños, Vicens-Vilanova, BarrantesVidal, Subirá y Obiols, (2007), quienes encontraron que son los adolescentes quienes reportan mayores conductas externalizadas probablemente por obtener una deseabilidad social en su grupo de pares.

Igualmente, con respecto a la diferencia de sexo, la mayoría de las adolescentes no presentan conductas externalizadas, mientras que un porcentaje pequeño, sí reporta dichas conductas, esto se debe a que las mujeres presentan una patología más internalizada presentando conductas tales como la depresión, problemas de relación y quejas somáticas (Lozano \& García, 2000), por su parte, los hombres presentan más comportamientos externalizados que las mujeres, como la búsqueda de atención y las conductas delictivas (Lozano \& García, 2000).

Por otra parte, de acuerdo al reporte dado por los padres, los adolescentes en su mayoría no presentan conductas externalizadas y solo una pequeña parte presenta dichas conductas. Los porcentajes bajos en los reportes de los padres frente a la presencia de las conductas externalizadas puede deberse a que para los padres las conductas internalizadas son más preocupantes que las externalizadas, debido a que estas conductas adolescentes resultan ser más perturbadoras en el contexto familiar (Medina, Navarro, Martinena, Baños, Vicens-Vilanova, Barrantes-Vidal, Subirá \& Obiols, 2007), de la misma manera, las relaciones afectivas pueden explicar dichos resultados, ya que estas generan seguridad, autoestima y fomentan el autoconcepto, generando en el adolescente destrezas emocionales, cognitivas y sociales que favorecen el comportamiento de los mismos (Silva \& Pillón, 2004).

Finalmente, se encuentran los reportes de los profesores quienes plantean que una muestra pequeña de los hombres no presenta conductas externalizadas mientras que el porcentaje restante y más significativo evidencia dichas conductas. Con respecto a las mujeres, los profesores reportan de igual manera, que solo una parte de la 
muestra no presenta conductas externalizadas, mientras que un porcentaje alto de las mujeres sí presenta dichas conductas, esto se debe a que las conductas externalizadas son más fáciles de detectar, puesto que van dirigidas hacia el exterior y las cuales afectan a otras personas, dentro de las conductas más frecuentes se encuentra la agresividad, la búsqueda de atención, ser irritable, entre otros (Achenbach y Rescorla, 2001); ello puede generar malestar en el interior del curso afectando significativamente el desarrollo de las clases. Además de esto, los profesores perciben un incremento en las conductas externalizadas debido al extenso tiempo que pasan estos con los adolescentes, sin embargo, la influencia ya sea positiva o negativa que tiene el grupo de pares o el contexto educativo en el cual se desarrolla el adolescente puede afectar significativamente dichos comportamientos (Edel, 2003).

Ahora bien, con respecto al rendimiento académico, la mayoría de la muestra de los adolescentes evidencia un nivel medio de rendimiento académico y solo una proporción se encuentra en un nivel alto o bajo de rendimiento académico, esto puede deberse a que los adolescentes presentan un tipo de rendimiento suficiente, lo cual indica que probablemente se basan principalmente en los conocimientos que les aporta el profesor durante la clase (Portellano, 1989), igualmente, habría que considerar para otro estudio la posibilidad de que estos resultados puedan estar asociados al comportamiento de los profesores, puesto que si son ellos quienes se encuentran desmotivados, su nivel de desempeño disminuirá afectando a los estudiantes (Cominetti y Ruiz, 1997). Claro está que el rendimiento académico puede afectarse también por la programación de actividades escolares, entendida como la manera en la cual los maestros logran planear las diferentes actividades escolares buscando la empatía de estas con los estudiantes y las temáticas (Brueckner y Bond, 1969, citados por Antoni, 2006). De acuerdo a esto, se evidencia que los adolescentes buscan aprobar el año escolar cumpliendo únicamente con las evaluaciones pedagógicas a través de las cuales se evalúa el logro, por lo que es fácil notar que estos adolescentes poseen un rendimiento de tipo suficiente (Antoni, 2006; Portellano, 1989).

A continuación se establecerán las asociaciones entre las variables estudiadas, es importante aclarar que ninguna de las relaciones establecidas arrojaron un nivel significativo de asociación, lo cual puede deberse a que la muestra con la cual se trabajó ha pasado por diversos programas de intervención en los últimos cinco años y ha participado en otras investigaciones, lo que pudo generar que los resultados obtenidos para el estudio no permitiesen establecer la significancia entre las variables objeto de investigación.

De acuerdo a lo anterior, se comenzará por analizar la asociación entre las variables funcionalidad familiar y las conductas externalizadas.

En cuanto a la asociación entre la Funcionalidad Familiar y el autorreporte que dan los adolescentes sobre las conductas externalizadas, no se encontró un nivel significativo de asociación, es decir que no necesariamente un adolescente con un nivel disfuncional dentro del núcleo familiar debe presentar conductas externalizadas, aunque la familia tiene ciertas implicaciones en cuanto al desarrollo de los adolescentes (Collins, 1995, Grotevant y Cooper, 1986; citados por Lerner y Steinberg, 2004), sin embargo, habría que considerar para un próximo estudio profundizar más sobre estas asociaciones, dado que otros autores han señalado que la función del núcleo familiar puede verse afectada por el estilo de crianza empleado por los padres de los adolescentes, ya que si el estilo es negligente, poco receptivo y poco exigente, puede generar en los adolescentes bajos niveles de individualización así como mayor riesgo de presentar problemas de conducta (Hoffman, Paris \& Hall, 1997), asimismo, las prácticas parentales utilizadas como forma de control psicológico se encuentran estrechamente asociadas a las conductas tanto internalizadas como externalizadas (Guevara et ál.,2007), donde estas últimas, son generadas por el estrés y la insatisfacción parental (Cabrera et ál., 2006).

Por otro lado, se estableció que no existe un nivel significativo de asociación entre la funcionalidad familiar y el rendimiento académico, es decir que un nivel disfuncional del núcleo familiar no es la única variable que pudiera explicar los niveles de rendimiento académico en los adolescentes que participaron en este estudio. Cabe resaltar la necesidad de profundizar más en esta asociación, dado que el instrumento empleado evalúa de una manera general la funcionalidad familiar y no es sensible a identificar aspectos más concretos del tipo de relación padres-hijos. Al respecto se podría entender que probablemente no son los niveles de funcionalidad los que afectan el rendimiento de los adolescentes sino tal vez el des- 
acuerdo constante que se presenta entre los padres y los adolescentes son los que generan el malestar en el interior del hogar, ya que los hijos durante la etapa de la adolescencia demandan importantes cambios en el sistema familiar (Comunian, 1996). De la misma manera, la familia se siente angustiada cuando sus hijos no obtienen notas satisfactorias o esperadas (Antoni, 2006), de igual manera, el estilo parental afecta el desempeño académico de los adolescentes, puesto que un estilo parental permisivo genera un nivel académico menor (Tilano et ál., 2008).

De acuerdo a los resultados arrojados y a que las asociaciones anteriores no tienen niveles significativos de asociación podría establecerse que no son los niveles de funcionalidad familiar los que interfieren directamente con las conductas externalizadas y el rendimiento académico, esto podría explicarse a través de las practicas parentales, puesto que pueden ser autoritarias, negligentes y permisivas (Solis et ál., 2006). Además de esto en un estudio realizado en Bogotá, Cabrera, Guevara y Barrera (2006), encontraron que las funciones como esposos y como padres y las prácticas parentales influyen significativamente en la aparición de las conductas externalizadas, de igual forma que el estrés y los niveles de satisfacción parental, fomentan las conductas externalizadas de los adolescentes. Estos aspectos podrían ser objeto de otra investigación que permitiera dilucidar más ampliamente dichas asociaciones.

Al igual que las anteriores asociaciones, no se encuentra un nivel significativo de asociación entre el rendimiento académico y las conductas externalizadas reportadas por los adolescentes, es decir, que la presencia o ausencia de las conductas externalizadas no afecta el rendimiento académico de los adolescentes, esto puede deberse a que las expectativas de la familia, de los docentes y de los mismos estudiantes pueden ser ventajosas y desventajosas a la vez, a la hora de evaluar el rendimiento académico, ya que estas se encuentran cargadas con prejuicios, actitudes y conductas que pueden generar mayor desempeño escolar pero a su vez pueden disminuirlo (Cominetti \& Ruiz 1997).

Finalmente, se sugiere para próximas investigaciones tener en cuenta que la muestra no esté siendo objeto de otras investigaciones, puesto que esto puede generar sesgo en los resultados de los instrumentos. Además de esto, se sugiere realizar un trabajo dirigido hacia las prác- ticas y tipos parentales ya que esta podría ser una variable que afectaría tanto las conductas externalizadas como el rendimiento académico.

\section{Referencias}

Achenbach, T. \& Rescorla, L. (2001a). Manual for the ASEBA School: Age Forms \& Profiles. Child Behavior Checklist for Ages 6-18, Teacher's report from, youth self-report and integrated system of multi-informant assessment. Burlington: University of Vermont.

Achenbach, T. \& Rescorla, L. (2001b). Manual for the ASEBA School- Age Forms \& Profiles. Burlington, VT: University of Vermont, Research Center for Children, Youth, \& families.

Antoni, M. (2006). Estrategias para mejorar el rendimiento académico de los adolescentes. Madrid: Pirámide.

Ballesteros, P. (1995). El conflicto marital y su relación con problemas de ajuste en los niños. Trabajo monográfico. Fundación Universitaria Konrad Lorenz

Barber, B. (2002). Intrusive Parenting. En How psychological control affects children and adolescents. Washington: American Psychological Association.

Briones, G. (1998). La investigación de la comunidad. Colombia: Tercer mundo.

Cabrera, V., Guevara, I. \& Barrera, F. (2006). Relaciones maritales, relaciones paternas y su influencia en el ajuste psicológico de los hijos. Acta Colombiana de Psicología. 9, 2. 115-126.

Cascón, I. (2000). Análisis de las calificaciones escolares como criterio de rendimiento académico. En red. Recuperado en: www3.usal.es/inicio/investigación/jornadas/jornada2/comunc/cl7.html

Clayton, R. R. (1992). Factores de riesgo y de protección frente al consumo de drogas: hacia un modelo explicativo del consumo de drogas en jóvenes de la CAPV. Instituto Deusto de Drogodependencias (Universidad de Deusto).

Cominetti, R. \& Ruiz, G. (1997). Algunos factores del rendimiento: las expectativas y el Género. Human Development Department.

Comunian, A. (1996). La relación entre la estructuras de la familia y la percepción de un modelo integrador; Informes Psicológicos.

Craig, G. (1997). Desarrollo psicológico. México: Prentice Hall. 
Edel, R. (2003). El rendimiento académico: concepto, investigación y desarrollo. Revista Electrónica Iberoamericana sobre Calidad, Eficacia y Cambio en Educación. 1, (2), 1-15.

Forero, L., Avendaño, M., Durán, M., Duarte, Z. \& Campo, A. (2006). Consistencia interna y análisis de factores de la escala APAGAR para evaluar el funcionamiento familiar en estudiantes de básica secundaria. Revista Colombiana de Psiquiatría. 1, (25), 23-29.

Graber, J. A. (2004). Internalizing Problems During Adolescence. En R. M. Lerner \& Steinberg, L. (Eds.). Handbook of a adolescent psychology 2." ed., (pp. 155-188). New York: Wiley.

Guevara, I., Cabrera, V. \& Barrera, F. (2007). Factores contextuales y emocionales morales como predictores del ajuste psicológico en la adolescencia. Universitas Psychologica. 6, (2), 269-283.

Hoffman, L. Paris, S. \& Hall, E. (1997). Psicología del desarrollo hoy. Madrid: McGraw-Hill.

Jiménez, M. (2000). Competencia social: intervención preventiva en la escuela. Universidad de Alicante. Infancia y Sociedad. 24. 21-48.

Jiménez, T., Musitu, G. \& Murgui, S. (2005). Familia, apoyo social y conducta delictiva en la adolescencia: efectos directos y mediadores. Anuario de Psicología. 36, (2), 559-579.

Lerner, R. \& Steinberg, L. (2004). Handbook of adolescent psychology. New Jersey: Wiley.

Lozano, L. \& García, E. (2002). El rendimiento escolar y los trastornos emocionales y comportamentales. Psicothema. 12, (2), 340-343.

Maestre, E., Moya, J., Edo, S., Mezquita, L., Rupérez, M. \& Villa, H. (2004). Relación de la personalidad y los factores de internalización y externalización en niños. Recuperado el 29 de abril de 2010 en www.uji.es/ bin/publ/edicions/jfi12/1.pdf

Medina, C., Navarro, B., Martinena, P., Baños, I., Vicens-Vilanova, J., Barrantes-Vidal, N., Subirá, S. \& Obiols, J. (2007). Evaluación de la conducta adolescente con las escalas de Achenbach: ¿Existe concordancia entre diferentes informantes?. Salud Mental. 30, (5), 33-39.

Mendoza, S., Soler, E., Sainz, L., Gil, A., Mendoza, H. F. \& Pérez, C. (2006). Análisis de la dinámica y funcionalidad familiar en atención primaria. Archivos en Medicina Familiar. 8, (1), 27-32.

Paz, S. (2007). Problemas en el desempeño escolar y su relación con su funcionalismo familiar en alumnos de EGB1. Revista de la Facultad de Medicina. 8, (1), 27-32.

Portellano, A. (1989). Fracaso escolar. Diagnóstico e intervención. Una perspectiva neuropsicológica. Madrid: Ciencias de la Educación Preescolar y Especial.

Rodrigo, M., Máiquez, M., García, M., Mendoza, R., Rubio, A., Martínez, A. \& Martín, J. (2004). Relaciones padres-hijos y estilos de vida en la adolescencia. Psicothema. 16, (2), 203-210.

Sattler, J. (2003). Evaluación infantil: aplicaciones cognitivas. México: Manual Moderno.

Smilkstein, G. (1978). The family APGAR: a proposal for a family function test and its use by physicians. $J$. Fam. Pract. 6, (6), 1231-1239.

Solis, L., Huerta, E., Vásquez, L., Alfaro, I., Sánchez, H. \& Hernández, C. (2006). análisis de la dinámica y funcionalidad familiar en atención primaria. Archivos en Medicina Familiar. 8, (1), 27-32.

Silva, I. \& Pillón, S. (2004). Factores protectores y de riesgo asociados al uso de alcohol en adolescentes hijos de padre alcohólico. Recuperado el 20 de agosto de 2010 en www.scielo.br/pdf/rlae/v12nspe/v12nspea10.pdf.

Tilano, L., Henao, G. \& Restrepo, J. (2008). Prácticas educativas y desempeño académico en adolescentes escolarizados en el grado noveno de instituciones educativas oficiales del municipio de Envigado. Revista El Ágora. 9, (1), 35-51.

Wicks-Nelson, R. (2000). Psicopatología del niño y el adolescente. Madrid: Prentice Hall. 